\title{
D-Lactate altered mitochondrial energy production in rat brain and heart but not liver
}

\author{
Binbing Ling ${ }^{1}$, Fei Peng ${ }^{1}$, Jane Alcorn ${ }^{1}$, Katharina Lohmann², Brian Bandy ${ }^{1}$ and Gordon A Zello ${ }^{1 *}$
}

\begin{abstract}
Background: Substantially elevated blood D-lactate (DLA) concentrations are associated with neurocardiac toxicity in humans and animals. The neurological symptoms are similar to inherited or acquired abnormalities of pyruvate metabolism. We hypothesized that DLA interferes with mitochondrial utilization of L-lactate and pyruvate in brain and heart.

Methods: Respiration rates in rat brain, heart and liver mitochondria were measured using DLA, LLA and pyruvate independently and in combination.

Results: In brain mitochondria, state 3 respiration was 53\% and 75\% lower with DLA as substrate when compared with LLA and pyruvate, respectively $(p<0.05)$. Similarly in heart mitochondria, state 3 respiration was $39 \%$ and $86 \%$ lower with DLA as substrate when compared with LLA or pyruvate, respectively $(p<0.05)$. However, state 3 respiration rates were similar between DLA, LLA and pyruvate in liver mitochondria. Combined incubation of DLA with LLA or pyruvate markedly impaired state 3 respiration rates in brain and heart mitochondria $(p<0.05)$ but not in liver mitochondria. DLA dehydrogenase activities were 61\% and 51\% lower in brain and heart mitochondria compared to liver, respectively, whereas LLA dehydrogenase activities were similar across all three tissues. An LDH inhibitor blocked state 3 respiration with LLA as substrate in all three tissues. A monocarboxylate transporter inhibitor blocked respiration with all three substrates.
\end{abstract}

Conclusions: DLA was a poor respiratory substrate in brain and heart mitochondria and inhibited LLA and pyruvate usage in these tissues. Further studies are warranted to evaluate whether these findings support, in part, the possible neurological and cardiac toxicity caused by high DLA levels.

Keywords: D-Lactate, Mitochondrial function, Rat, Brain, Heart

\section{Introduction}

Lactate exists as two stereoisomers, L-lactate and D-lactate. Under healthy physiological conditions, L-lactate is the major enantiomer found in blood whereas D-lactate is normally present in very low concentrations [1]. However, supra-physiological levels of D-lactate have been found in several disease states such as diarrhea, short bowel syndrome, and diabetes [2,3]. Most research in this area focus on the cause and the consequences of extremely high levels of D-lactate (> $3 \mathrm{mM}$ D-lactate in plasma, resulting in D-lactic acidosis) in the body [3-7]. Although sub-clinical levels of D-lactate (high D-lactate

\footnotetext{
* Correspondence: Gordon.Zello@usask.ca

${ }^{1}$ College of Pharmacy and Nutrition, University of Saskatchewan, Saskatoon, SK, Canada

Full list of author information is available at the end of the article
}

levels, no acidosis) have been reported in several chronic diseases including diabetes and chronic fatigue syndrome [8,9], few studies explore the potential negative outcomes of such sub-clinical concentrations of D-lactate circulation in the body [10]. Interestingly, the clinical symptoms due to high levels of D-lactate (D-lactic acidosis) are similar to inherited or acquired abnormalities of pyruvate metabolism [11]. Therefore, D-lactate may directly or indirectly interfere pyruvate metabolism pathways, which are essential for mitochondrial energy production [12]. Any disturbance in pyruvate metabolism pathways may eventually impair mitochondrial energy generation and thus affect organs that are more highly energy dependent $[13,14]$.

The brain and heart are metabolically active organs with substantial energy requirements. The major cellular
C Biomed Central

() 2012 Ling et al; licensee BioMed Central Ltd. This is an Open Access article distributed under the terms of the Creative Commons Attribution License (http://creativecommons.org/licenses/by/2.0), which permits unrestricted use, distribution, and reproduction in any medium, provided the original work is properly cited. 
pathways of energy production are glycolysis and mitochondrial oxidative phosphorylation [15]. During glycolysis, glucose is converted to pyruvate, which is accompanied by the production of ATP and NADH [15]. In mammalian cells, the enzymes responsible for pyruvate metabolism are located in the mitochondria [16]. Thus, pyruvate generated during glycolysis is transported into the mitochondria via monocarboxylate transporters (MCTs) particularly MCT1 [17]. In the mitochondria, pyruvate breakdown irreversibly funnels the products of glycolysis into the Krebs cycle to produce ATP and a large quantity of NADH [12]. NADH produced by both processes is then used to fuel mitochondrial ATP synthesis via oxidative phosphorylation or mitochondrial respiratory chain phosphorylation $[15,16]$.

In some tissues, L-lactate oxidation can provide cellular energy in addition to glycolysis $[18,19]$. For example, L-lactate has been identified as the preferential oxidative energy substrate for the brain during excitation [20]. The Astrocyte-Neuron Lactate Shuttle hypothesis suggests that fuel for increased energy requirement of neurons during excitation is supplied by L-lactate from the surrounding astrocytes rather than glucose [18,21]. Following its transport into the cell, cytosolic L-lactate is converted to pyruvate by L-lactate dehydrogenase (LDH), an enzyme using NAD as a cofactor [18], which subsequently enters the TCA cycle in the mitochondria for further energy production. In addition, mitochondria also contain significant amounts of LDH, located largely in the inter-membrane space [18]. L-lactate transported into the mitochondria via MCTs is metabolized to pyruvate for energy production. Therefore, mitochondrial utilization of both L-lactate and pyruvate is crucial for cellular bioenergetics.

D-Lactate, recognized by MCTs [22], can competitively inhibit L-lactate and/or pyruvate transport via MCTs at the cellular level. For example, D-lactate inhibited L-lactate uptake into erythrocytes and brain cells and pyruvate uptake into cardiac myocytes [23,24]. Once D-lactate has entered the cells, it can affect the transport of L-lactate and/or pyruvate into the mitochondria and thus affects the usage of pyruvate and/or lactate. In fact, D-lactate interference of pyruvate metabolism has been postulated based on the clinical similarities between D-lactic acidosis and inherited or acquired abnormalities of pyruvate metabolism [25]; however, no experimental data are available to support this inference.

Cellular D-lactate is metabolized by mitochondrial Dlactate dehydrogenase (DDH) using FAD as cofactor $[26,27]$. The expression of DDH is tissue dependent and therefore affects the usage of D-lactate in different tissues $[26,27]$. For example, due to low expression levels of DDH in the brain, the rate of oxidation of D-lactate in the brain is considerably slower compared to that of L-lactate $[26,27]$. D-Lactate accumulation, then, may compromise energy metabolism by interfering with the mitochondrial usage of its more efficient energy substrates pyruvate and/or L-lactate and thus lead to toxicity. Energy deficiency in the brain of chickens has been reported following intracerebral infusion of D-lactate which suggests D-lactate's involvement in altered substrate utilization and ATP generation [28]. In this study, we hypothesized that D-lactate interferes with mitochondrial utilization of L-lactate and pyruvate.

The objectives of this study were to compare the mitochondrial utilization of D-lactate to L-lactate and pyruvate in mitochondria from rat heart, liver and brain tissues. We also investigated the effects of D-lactate on mitochondrial respiration with L-lactate or pyruvate as substrates. The D- and L-lactate dehydrogenase activities from different tissue mitochondria were also measured. To test the role of LDH on the mitochondrial respiration of L-lactate, a LDH inhibitor, oxamate (OX) was used in rat liver and brain. Our investigations also employed an MCT inhibitor, $\alpha$-cyano-4-hydroxycinnamate (CINN), to identify the role of mitochondrial MCTs in D-lactate, L-lactate and pyruvate metabolism in rat liver and brain.

\section{Materials and methods}

\section{Animals and Chemicals}

Male Wistar rats were obtained from Charles River Canada (St. Constant, PQ) and were housed in a temperature and humidity controlled facility $\left(22^{\circ} \mathrm{C} \pm 2^{\circ} \mathrm{C}\right)$ on a 12-hour light: dark cycle $(0700 \mathrm{~h}-1900 \mathrm{~h})$. Rats had free access to food and water and were allowed a 7day acclimatization period. Rats were provided a nutritionally adequate rat diet (Prolab ${ }^{\circledR}$ RMH 3000, Purina, Inc., Richmond, IN) ad libitum. This work was approved by the University of Saskatchewan's Animal Research Ethics Board, and adhered to the Canadian Council on Animal Care guidelines for humane animal use. Chemicals, unless specified, were purchased from SigmaAldrich.

\section{Mitochondrial Isolation}

Mitochondria were isolated from rat (250-300 g body weight, $\mathrm{n}=6$ ) brain, heart and liver following humane euthanasia (isoflurane anaesthesia and exanguination) and rapid removal of organs. Briefly, freshly isolated organs were homogenized using a glass-teflon homogenizer (brain and liver) or a Polytron homogenizer (heart) with an isolation medium containing $250 \mathrm{mM}$ sucrose, $10 \mathrm{mM}$ HEPES and $1 \mathrm{mM}$ EGTA (pH 7.2). In the case of brain, the mitochondria were isolated in the presence of $0.1 \%$ fat-free bovine serum albumin (BSA). 
The homogenate was then centrifuged at $1000 \mathrm{~g}$ for 8 min at $4^{\circ} \mathrm{C}$. The supernatant was collected and centrifuged at $10000 \mathrm{~g}$ for $10 \mathrm{~min}$ at $4^{\circ} \mathrm{C}$. The pellet was collected and washed twice with washing medium containing $250 \mathrm{mM}$ sucrose, $10 \mathrm{mM}$ HEPES and 0.1 mM EGTA. The final pellets of mitochondria were suspended in $2 \mathrm{~mL}$ of isolation medium without EGTA [29-32].

\section{Oxygen Uptake Studies}

Mitochondrial protein was measured as described by the biuret method using bovine serum albumin as standard [33]. Respiration rates of isolated mitochondria were measured with a Clark-type electrode (DW1; Hansatech Instruments Ltd, Norfolk, England) in a water-jacketed glass chamber with magnetic stirring. An oxygen electrode was used and the respiration chamber was kept constant at $30^{\circ} \mathrm{C}$. Oxygen uptake measurements were carried out in $1 \mathrm{~mL}$ of medium containing $210 \mathrm{mM}$ mannitol, $70 \mathrm{mM}$ sucrose, $0.1 \mathrm{mM}$ EDTA, $20 \mathrm{mM}$ Tris $/ \mathrm{HCl}, 3 \mathrm{mM} \mathrm{MgCl}_{2}, 5 \mathrm{mM}$ $\mathrm{KH}_{2} \mathrm{PO}_{4} / \mathrm{K}_{2} \mathrm{HPO}_{4}$ and $0.2 \%$ BSA (pH 7.4) [34]. Assays were performed in duplicate using fresh mitochondria. The respiratory parameters of the mitochondria were tested using a respiratory cocktail (containing $62.5 \mu \mathrm{M}$ each of malate, glutamate, alpha-ketoglutarate and pyruvate), pyruvate + malate, L-lactate + malate, or D-lactate + malate. Pyruvate $(10 \mathrm{mM})$ and $\mathrm{D} / \mathrm{L}$-lactate $(5$ $\mathrm{mM}$ ) were added alone or in combination. To inhibit the mitochondrial monocarboxylate transporter (mMCT), $5 \mathrm{mM} \alpha$-cyano-4-hydroxycinnamate (CINN) was used. To inhibit mitochondrial LDH, $50 \mathrm{mM}$ oxamate (OX) was used. Respiration was initiated by the addition of $1 \mathrm{mg}$ protein of the mitochondrial suspension to the reaction medium, and a conventional respiratory experiment with transitions from state 4 to 3 was performed.

Mitochondrial respiratory state 4 is the resting state which is differentiated by relatively slow oxygen uptake and no availability of ADP [35]. On the other hand, mitochondrial respiratory state 3 is the active state with high rates of oxygen uptake and sufficient ADP supply [35]. Thus, state 3 was initiated by adding ADP (final concentration $0.1 \mathrm{mM}$ ). Mitochondrial function was assessed by the respiratory control ratio (RCR) and ADP:O ratios [36]. These two parameters are well accepted as indicators of electron transport chain coupling to ATP synthesis and efficiency of oxidative phosphorylation in the presence of different substrates. The RCR values were calculated as the ratio of the respiratory rate in state 3 , after addition of ADP, to the rate of oxygen uptake without ADP (state 4). The ratio between the amount of ADP phosphorylated and oxygen consumed (ADP/O ratio) was also calculated [37].

\section{L- and D-Lactate Dehydrogenase (LDH and DDH) Assay}

The LDH and DDH assays were performed photometrically by means of a 96 well spectrophotometer at 600 $\mathrm{nm}$ [38] at $25^{\circ} \mathrm{C}$. Briefly, the mitochondrial sample was incubated for $2 \mathrm{~min}$ in $2 \mathrm{~mL}$ of standard medium consisting of $0.2 \mathrm{mM}$ sucrose, $10 \mathrm{mM} \mathrm{KCl}, 20 \mathrm{mM}$ Hepes/ Tris, $\mathrm{pH} 7.2,1 \mathrm{mM} \mathrm{MgCl}$ in the presence of $30 \mu \mathrm{M}$ phenazine methosulphate (PMS) and $50 \mu \mathrm{M}$ dichloroindophenol (DCIP). DDH activity was determined by measuring the decrease in absorbance at $600 \mathrm{~nm}\left(\mathrm{~A}_{600}\right)$ due to DCIP reduction when $15 \mathrm{mM}$ D-lactate was added. The activity was expressed as nmol of DCIP reduced per min per mg of protein.

\section{Statistics}

Results are presented as mean \pm SEM. Respiration rates and RCR values were compared using one way ANOVA with Fisher's least significant difference (LSD) post hoc tests. The statistical differences between the values with the presence of different substrates and the values without addition of any of the test substrates are reported in tables (Table 1, 2 and 3). Due to the complexity, the rest of the statistical results are described in the results section rather than in the tables.

\section{Results}

Pyruvate, D-lactate or L-lactate as substrate for mitochondrial respiration

To demonstrate the ability of rat mitochondria from brain, liver and heart to use pyruvate, L-lactate or D-

Table 1 Respiratory parameters ${ }^{\mathrm{a}}$ of isolated rat liver mitochondria with pyruvate, D-lactate, or L-lactate or as combination.

\begin{tabular}{ccccc}
\hline Substrate $^{\mathbf{b}}$ & State $\mathbf{4}$ & State $\mathbf{3}$ & RCR & ADP/O \\
\hline No substrate & $3.24 \pm 0.28$ & $3.37 \pm 0.34$ & $1.04 \pm 0.02$ & \\
Cocktail $^{\mathrm{C}}$ & $4.11 \pm 0.13$ & $30.12 \pm 2.02^{*}$ & $7.33 \pm 0.46^{*}$ & $2.87 \pm 0.09$ \\
Pyruvate & $5.01 \pm 0.56$ & $19.17 \pm 2.56^{*}$ & $3.78 \pm 0.11^{*}$ & $2.56 \pm 0.04$ \\
D-lactate & $3.82 \pm 0.24$ & $14.11 \pm 1.13^{*}$ & $3.69 \pm 0.11^{*}$ & $2.47 \pm 0.11$ \\
L-lactate & $4.22 \pm 0.19$ & $15.02 \pm 0.98^{*}$ & $3.56 \pm 0.15^{*}$ & $2.30 \pm 0.24$ \\
Pyruvate + LLA & $6.20 \pm 0.41$ & $21.40 \pm 1.59^{*}$ & $3.45 \pm 0.06^{*}$ & - \\
Pyruvate + DLA & $3.86 \pm 0.23$ & $17.58 \pm 2.21^{*}$ & $4.56 \pm 0.51^{*}$ & - \\
LLA + DLA & $3.76 \pm 0.21$ & $11.78 \pm 0.41$ & $3.14 \pm 0.07^{*}$ & - \\
\hline
\end{tabular}

${ }^{a}$ Respiratory rates in $\mathrm{nmol} \mathrm{O}_{2} / \mathrm{mg}$ mitochondrial protein per min.

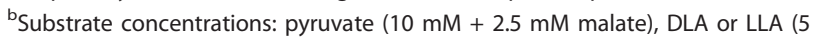
$\mathrm{mM}+2.5 \mathrm{mM}$ malate), and LLA+DLA (2.5 mM LLA and $2.5 \mathrm{mM}$ DLA+ $2.5 \mathrm{mM}$ malate).

${ }^{c}$ Cocktail contains $62.5 \mu \mathrm{M}$ each of malate, glutamate, alpha-ketoglutarate and pyruvate.

State 4: The respiration state without ADP.

State 3: The respiration state with the addition of ADP.

$\mathrm{RCR}$, Respiratory Control Ratio (state 3 rate:state 4 rate).

ADP/O ratio: The ratio between the amount of ADP phosphorylated and oxygen consumed.

*Significantly different from the values without substrate. Values were compared using one way ANOVA with LSD as post hoc test, $\alpha<0.05$. 
Table 2 Respiratory parameters ${ }^{\mathrm{a}}$ of isolated rat brain mitochondria with pyruvate, D-lactate, or L-lactate or as combination.

\begin{tabular}{ccccc}
\hline Substrate $^{\mathbf{b}}$ & State $\mathbf{4}$ & State $\mathbf{3}$ & RCR & ADP/O \\
\hline No substrate & $3.56 \pm 0.31$ & $5.21 \pm 0.14$ & $1.49 \pm 0.11$ & \\
Cocktail $^{\mathrm{C}}$ & $4.51 \pm 0.49$ & $29.43 \pm 2.92^{*}$ & $6.61 \pm 0.73^{*}$ & $2.92 \pm 0.01$ \\
Pyruvate & $3.59 \pm 0.21$ & $30.98 \pm 2.42^{*}$ & $8.46 \pm 0.55^{*}$ & $2.12 \pm 0.15$ \\
D-lactate & $3.18 \pm 0.16$ & $7.64 \pm 0.56$ & $2.39 \pm 0.10$ & - \\
L-lactate $^{3}$ & $3.18 \pm 0.16$ & $16.13 \pm 0.49^{*}$ & $4.66 \pm 0.17^{*}$ & $1.88 \pm 0.10$ \\
Pyruvate + LLA & $3.16 \pm 0.45$ & $22.09 \pm 4.42^{*}$ & $7.16 \pm 1.57^{*}$ & - \\
Pyruvate + DLA & $4.07 \pm 0.51$ & $16.41 \pm 2.85$ & $3.94 \pm 0.40$ & - \\
LLA + DLA & $3.40 \pm 0.34$ & $5.41 \pm 0.48$ & $1.60 \pm 0.08$ & - \\
\hline
\end{tabular}

${ }^{a}$ Respiratory rates in $\mathrm{nmol} \mathrm{O}_{2} / \mathrm{mg}$ mitochondrial protein per min.

${ }^{b}$ Substrate concentrations: pyruvate $(10 \mathrm{mM}+2.5 \mathrm{mM}$ malate), DLA or LLA (5 $\mathrm{mM}+2.5 \mathrm{mM}$ malate), and LLA+DLA (2.5 mM LLA and $2.5 \mathrm{mM}$ DLA+ $2.5 \mathrm{mM}$ malate).

${ }^{c}$ Cocktail contains 62.5 meach of malate, glutamate, alpha-ketoglutarate and pyruvate.

State 4: The respiration state without ADP.

State 3: The respiration state with the addition of ADP.

RCR: Respiratory Control Ratio (state 3 rate:state 4 rate).

ADP/O ratio: The ratio between the amount of ADP phosphorylated and oxygen consumed.

*Significantly different from the values without substrate. Values were compared using one way ANOVA with LSD as post hoc test, $\alpha<0.05$.

lactate as substrates, oxygen uptake was measured under respiratory state 4 conditions and state 3 conditions in the presence of $0.1 \mathrm{mM}$ ADP. Mitochondria isolated from brain, heart and liver readily oxidized pyruvate and L-lactate with a respiration control ratios (RCR) values higher than 5 with a respiratory substrate cocktail and

Table 3 Respiratory parameters ${ }^{a}$ of isolated rat heart mitochondria with pyruvate, D-lactate, L-lactate alone or as combination as substrates.

\begin{tabular}{ccccc}
\hline Substrate $^{\mathbf{b}}$ & State $\mathbf{4}$ & State $\mathbf{3}$ & RCR & ADP/O \\
\hline No substrate & $3.07 \pm 0.15$ & $7.72 \pm 0.55$ & $2.52 \pm 0.08$ & \\
Cocktail $^{c}$ & $11.35 \pm 1.52^{*}$ & $70.89 \pm 5.89^{*}$ & $6.39 \pm 0.59^{*}$ & $2.76 \pm 0.02$ \\
Pyruvate & $12.51 \pm 1.19^{*}$ & $74.74 \pm 6.22^{*}$ & $5.95 \pm 0.22^{*}$ & $3.01 \pm 0.02$ \\
D-lactate & $4.62 \pm 0.19$ & $9.27 \pm 1.31$ & $2.01 \pm 0.27$ & - \\
L-lactate & $4.58 \pm 0.58$ & $15.17 \pm 2.67$ & $3.24 \pm 0.21$ & $2.11 \pm 0.34$ \\
Pyruvate + LLA & $4.30 \pm 0.25$ & $18.23 \pm 1.52$ & $4.23 \pm 0.15^{*}$ & - \\
Pyruvate + DLA & $5.28 \pm 0.42$ & $15.03 \pm 0.74$ & $2.91 \pm 0.21$ & - \\
LLA + DLA & $3.57 \pm 0.22$ & $3.95 \pm 0.26$ & $1.11 \pm 0.08$ & - \\
\hline
\end{tabular}

${ }^{a}$ Respiratory rates in $\mathrm{nmol} \mathrm{O}_{2} / \mathrm{mg}$ mitochondrial protein per min.

bSubstrate concentrations: pyruvate (10 mM $+2.5 \mathrm{mM}$ malate), DLA or LLA (5 $\mathrm{mM}+2.5 \mathrm{mM}$ malate), and LLA+DLA (2.5 mM LLA and $2.5 \mathrm{mM}$ DLA+ $2.5 \mathrm{mM}$ malate).

${ }^{c}$ Cocktail contains 62.5 ueach of malate, glutamate, alpha-ketoglutarate and pyruvate.

State 4: The respiration state without ADP.

State 3: The respiration state with the addition of ADP.

RCR: Respiratory Control Ratio (state 3 rate:state 4 rate).

ADP/O ratio: The ratio between the amount of ADP phosphorylated and oxygen consumed.

* Significantly different from the values without substrate. Values were compared using one way ANOVA with LSD as post hoc test, $\alpha<0.05$.
ATP/O values higher than 2 , which are the generally accepted as normal for carefully prepared mitochondria from liver, heart and brain (Tables 1, 2 and 3). However, mitochondria isolated from brain and heart did not oxidize D-lactate efficiently as demonstrated by low state 3 respiration rates and RCR values close to values recorded without substrate, as well as unmeasurable $\mathrm{ADP} / \mathrm{O}$ ratios ( $\mathrm{p}>0.05$, Table 2 and 3 ). Liver mitochondria oxidized D-lactate with a RCR of 3.67 and ADP/O ratio of 2.47 (Table 2), which is similar to the L-lactate and pyruvate $(\mathrm{p}>0.05)$. When compared to brain and liver, mitochondria from heart had twice as high or greater state 3 and state 4 respiration when using a cocktail or pyruvate $(\mathrm{p}<0.05)$, but not L-lactate. Pyruvate showed the highest RCR in brain mitochondria with the lowest in liver (brain $>$ heart $>$ liver, $\mathrm{p}<0.05$ ). The RCR values for L-lactate in brain mitochondria were significantly higher than that of the liver and heart mitochondria $(\mathrm{p}<0.05)$.

\section{Effect of D-lactate or L-lactate on mitochondrial oxidation} of pyruvate in liver, heart and brain

D-Lactate significantly impaired oxygen consumption caused by pyruvate in rat brain and heart mitochondria. The oxygen consumption rate inhibition caused by Dlactate was slightly stronger compared to L-lactate (e.g. $47 \%$ ( $\mathrm{p}<0.05)$ vs. $29 \%$ ( $\mathrm{p}>0.05$ ) reduction in state 3 in the brain, respectively compared to pyruvate alone as substrate). Interestingly, co-administration of D-lactate or L-lactate did not change mitochondrial respiration using pyruvate as substrate in liver mitochondria ( $\mathrm{p}>$ $0.05)$. In addition, D-lactate significantly decreased oxygen consumption caused by L-lactate in rat brain $(67 \%$ reduction in state $3, \mathrm{p}<0.05)$ and heart $(74 \%$ reduction in state $3, \mathrm{p}<0.05)$ mitochondria with a slight but nonsignificant change in liver $(22 \%$ reduction in state $3, \mathrm{p}>$ 0.05) mitochondria.

\section{DDH and LDH activities in brain, heart and liver mitochondria}

DDH activity was significantly higher in rat liver mitochondria compared to rat brain and heart mitochondria (Figure 1). DDH activity in rat liver mitochondria was similar to LDH activity. The activity of LDH was similar in the brain, heart and liver mitochondria.

\section{Effect of oxamate (OX) and $\alpha$-cyano-4-hydroxycinnamate (CINN) on mitochondrial respiration using pyruvate, D- lactate and L-lactate}

In the presence of OX, a known LDH inhibitor, the oxygen consumption rate in state 3 and the RCR in brain and liver mitochondria were similar to the values without substrates (Table 4). In other words, mitochondrial L-lactate oxidation was completely blocked by OX. It is 


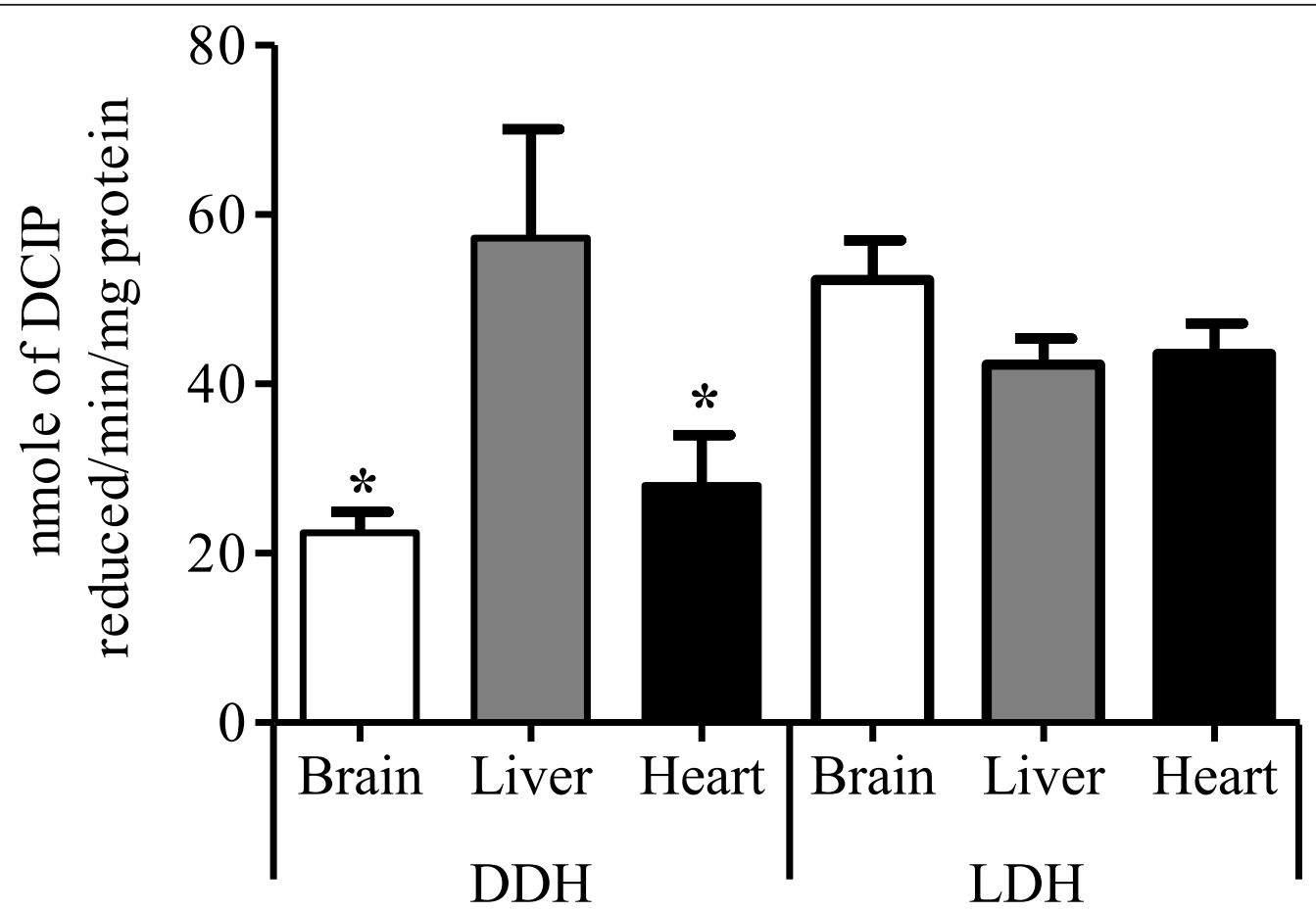

Figure 1 The enzyme activities of DDH and LDH expressed as nmole of dichloroindophenol (DCIP) reduced/min/mg protein (Mean \pm SEM) in mitochondria from rat brain, liver and heart $(\mathbf{n}=6)$. * Different from liver mitochondria, $P<0.05$.

important to note that OX also partially inhibited respiration with pyruvate $(\mathrm{p}<0.05)$ and $\mathrm{D}$-lactate $(\mathrm{p}>$ 0.05 ) (e.g. $42 \%$ and $37 \%$ decrease respectively in state 3 in the brain) as substrate, which suggests that OX had other effects than just inhibition of LDH. In the

Table 4 Respiratory parameters ${ }^{\mathrm{a}}$ of isolated rat brain and liver mitochondria with pyruvate, D-lactate, L-lactate as substrates in the presence of oxamate, a LDH inhibitor.

\begin{tabular}{ccccc}
\hline Substrate $^{\mathbf{b}}$ & Tissue & State $\mathbf{4}$ & State $\mathbf{3}$ & $\mathbf{R C l}$ \\
\hline Pyruvate + OX & Brain & $3.20 \pm 0.57$ & $17.96 \pm 2.51$ & $5.74 \pm 0.41$ \\
& Liver & $2.61 \pm 0.09$ & $9.98 \pm 0.31$ & $3.83 \pm 0.05$ \\
LLA + OX & Brain & $3.45 \pm 0.12$ & $4.27 \pm 0.35$ & $1.23 \pm 0.06$ \\
& Liver & $3.28 \pm 0.23$ & $4.19 \pm 1.08$ & $1.25 \pm 0.23$ \\
DLA + OX & Brain & $3.25 \pm 0.15$ & $4.83 \pm 1.11$ & $1.47 \pm 0.30$ \\
& Liver & $4.11 \pm 0.15$ & $14.34 \pm 0.21$ & $3.50 \pm 0.12$ \\
Pyr +LLA+ OX & Brain & $3.06 \pm 0.42$ & $7.14 \pm 1.23$ & $2.32 \pm 0.14$ \\
& Liver & $2.16 \pm 0.22$ & $2.73 \pm 0.45$ & $1.29 \pm 0.25$ \\
\hline
\end{tabular}

${ }^{\mathrm{a}}$ Respiratory rates in $\mathrm{nmol} \mathrm{O}_{2} / \mathrm{mg}$ mitochondrial protein per min.

${ }^{b}$ Substrate concentrations: pyruvate $(10 \mathrm{mM}+2.5 \mathrm{mM}$ malate) and LLA or DLA (5 mM + $2.5 \mathrm{mM}$ malate); OX, oxamate (50 mM).

State 4: The respiration state without ADP.

State 3: The respiration state with the addition of ADP.

RCR: Respiratory Control Ratio (state 3 rate:state 4 rate).

ADP/O ratio: The ratio between the amount of ADP phosphorylated and oxygen consumed.

*Significantly different from the values without substrate. Values were compared using one way ANOVA with LSD as post hoc test. $\alpha<0.05$

Note: Refer to Table 1 and 2 for the respiratory parameters without substrates or with cocktail. presence of CINN, a known MCT inhibitor, the respirations caused by pyruvate, L-lactate and D-lactate were completely blocked in brain mitochondria with the state 3 , state 4 respiration rate and RCR similar to values in the absence of substrate (Table 5).

Table 5 Respiratory parameters ${ }^{a}$ of isolated rat brain and liver mitochondria with pyruvate, D-lactate, L-lactate as substrates in the presence of CINN, a monocarboxylate transporter inhibitor.

\begin{tabular}{ccccc}
\hline Substrate $^{\mathbf{b}}$ & Tissue & State 4 & State 3 & RCI \\
\hline Pyruvate + CINN & Brain & $3.42 \pm 0.33$ & $5.88 \pm 0.72$ & $1.72 \pm 0.13$ \\
& Liver & $5.16 \pm 0.19$ & $10.08 \pm 0.74$ & $1.97 \pm 0.21$ \\
LLA + CINN & Brain & $2.87 \pm 0.04$ & $3.83 \pm 0.52$ & $1.34 \pm 0.20$ \\
& Liver & $2.89 \pm 0.29$ & $3.68 \pm 0.15$ & $1.32 \pm 0.20$ \\
DLA + CINN & Brain & $2.46 \pm 0.29$ & $2.79 \pm 0.23$ & $1.16 \pm 0.23$ \\
& Liver & $2.50 \pm 0.40$ & $2.52 \pm 0.16$ & $1.03 \pm 0.10$ \\
\hline
\end{tabular}

${ }^{\mathrm{a}}$ Respiratory rates in $\mathrm{nmol} \mathrm{O}_{2} / \mathrm{mg}$ mitochondrial protein per min.

${ }^{b}$ Substrate concentrations: pyruvate $(10 \mathrm{mM}+2.5 \mathrm{mM}$ malate) and LLA or DLA ( $5 \mathrm{mM}+2.5 \mathrm{mM}$ malate); CINN, $\alpha$-cyano-4-hydroxycinnamate (5 mM). State 4: The respiration state without ADP.

State 3: The respiration state with the addition of ADP. $\mathrm{RCR}$, Respiratory Control Ratio (state 3 rate:state 4 rate). ADP/O ratio: The ratio between the amount of ADP phosphorylated and oxygen consumed.

*Significantly different from the values without substrate. Values were compared using one way ANOVA with LSD as post hoc test. $\alpha<0.05$ Note: Refer to Table 1 and 2 for the respiratory parameters without substrates or with cocktail. 


\section{Discussion}

D-lactate is present at low levels in the body and can be well utilized by the liver in both human and animal under healthy conditions [39]. However, elevated levels of blood D-lactate in several disease states $[5,40]$ can result in D-lactate accumulation in specific tissues and potential toxicity. In fact, high D-lactate levels are associated with neurological and cardiac dysfunction $[7,41]$. The underlying mechanisms of such toxicities are not fully understood. In our study, we examined whether Dlactate was an efficient energy substrate for brain and heart mitochondrial and, if not, whether it could interfere with mitochondrial utilization of two major cellular energy substrates, L-lactate and pyruvate. Such interference could result in a cellular energy deficiency and, therefore, begin to explain, in part, the neurological and cardiac toxicities observed with D-lactic acidosis.

The majority of cellular ATP is generated by glycolysis and oxidative phosphorylation of pyruvate, the latter of which takes place within the mitochondria of eukaryotic cells. Impaired ATP production in mitochondria can lead to cellular energy deficiency and eventually organ dysfunctions [42]. To understand the factors that may influence mitochondrial pyruvate metabolism, isolated mitochondria are often used with measurements of respiration states, RCR and ADP/O ratios. Mitochondrial integrity and functionality was assessed with the supply of optimal substrates to assure reliable outcomes [30]. The high degree of coupling with high $\mathrm{RCR}(>6)$ and a ADP/O ratio close to 3 using a typical cocktail solution (Table 1, 2, 3) demonstrated that the isolated mitochondria were functionally well preserved $[43,44]$. Malate was also added into our reaction mixture as it is a vital cofactor for various mitochondrial substrate transporters. It is essential for mitochondrial respiration and ensures a continuous flow of substrate across mitochondrial membranes into the matrix. Without the presence of malate, the respiration rate for state 3 is not induced by the addition of ADP with any of the selected substrates. Although mitochondria contain malic enzyme [45], malate alone did not support mitochondrial respiration in all three tissues (data not shown), which is consistent with the literature $[46,47]$.

Although pyruvate is a key substrate for mitochondrial respiration, L-lactate can serve as a preferential substrate for mitochondrial respiration, particularly in highly metabolic tissues under conditions that have increased requirement for mitochondrial respiration [48]. In our study, ADP addition stimulated mitochondrial electron transport chain activity and oxygen consumption in all mitochondrial preparations as indicated by higher mitochondrial state 3 respiration and RCR in the presence of either L-lactate or pyruvate. Therefore, both pyruvate and L-lactate are well oxidized in rat brain, heart and liver mitochondria (Table 1, 2, 3). The heart demonstrated the highest state 3 and state 4 respiration rates with pyruvate and L-lactate as compared with brain and liver. This was expected since heart mitochondria has higher oxidative capacity compared to brain and liver [49].

RCR values can indicate the efficiency of electron transport chain coupling activity with oxidative phosphorylation [50]. Brain and heart, but not liver, mitochondria demonstrated lower RCR ratios (less than 3) with D-lactate as substrate compared to pyruvate or Llactate (Table 2 and 3). D-Lactate also reduced oxygen consumption rates in brain and heart mitochondria but not liver and impeded efficient utilization of pyruvate and L-lactate by brain and heart mitochondria. These data coupled with findings of limited D-lactate dehydrogenase activity in brain and heart mitochondria (Figure 1) suggest that D-lactate is a poor mitochondrial respiration substrate in these tissues. Relatively high Dlactate dehydrogenase activities in liver mitochondria likely maintain efficient oxidative phosphorylation in these mitochondria in essence counteracting the effects of D-lactate noted in brain and heart mitochondria. Therefore, an accumulation of D-lactate in the brain and heart tissue may represent a secondary disorder of mitochondrial function by interfering with L-lactate and pyruvate metabolism. This has been postulated by other researchers and our study provides the first supportive experimental data for this postulate to our knowledge $[28,51]$.

Interestingly, we also observed changes in oxygen uptake by pyruvate in rat heart, brain and liver mitochondria in the presence of L-lactate though the extents of inhibition were less compared to D-lactate (Table 1, $2,3)$. Both D and L-lactate share the same mitochondrial membrane transporter and have the potential to competitively inhibit pyruvate transport into the mitochondria for energy production [28]. In the liver, both isomers of lactate can be recognized by mitochondrial lactate dehydrogenase (LDH and DDH respectively) and converted into pyruvate. Such conversion can therefore compensate for the reductions in pyruvate concentrations in the mitochondria resulting from inhibition of pyruvate transport into this organelle $[52,53]$. However, the degree of the compensation depends on the enzyme tissue distribution and activity. In our study, DDH activities were significantly lower in rat brain and heart mitochondria compared to liver where as LDH activities were similar between all three tissues (Figure 1). The low level of DDH in rat brain and heart may explain the strong inhibition of D-lactate on mitochondrial 
respiration rates using pyruvate as substrate in these two tissues.

To further investigate these findings, an LDH inhibitor (oxamate) was used to block lactate oxidation in rat brain and liver mitochondria [53]. In the presence of oxamate, mitochondrial respiration was maintained with pyruvate as substrate while mitochondrial respiration was reduced with administration of L-lactate or coadministration of L-lactate with pyruvate. These data suggest LDH plays an important role in the oxidation of L-lactate in isolated mitochondria. To investigate the possible mechanism of L- and D-lactate mediated inhibition of pyruvate metabolism, a monocarboxylate transporter (MCT) inhibitor, CINN, was used to inhibit MCT function. Marked reduction of mitochondrial respiration rates in the presence of CINN suggests a role for MCTs in the mitochondrial uptake of both isomers of lactate and pyruvate. Competitive interactions occurring at MCT may explain the inhibitory effect of D-lactate on L-lactate and pyruvate.

In conclusion, our study identified that D-lactate is a poor substrate for rat brain and heart mitochondria, but an efficient substrate for liver mitochondrial respiration. Low levels of DDH activity in rat brain and heart likely explain its poor utilization by mitochondria of these tissues. Additionally, D-lactate inhibited brain and heart mitochondrial respiration caused by pyruvate and L-lactate. L-Lactate also inhibited pyruvate induced mitochondrial respiration in liver, brain and heart but could maintain heart and brain mitochondrial respiration via LDH mediated conversion of L-lactate to pyruvate. Furthermore, an inhibitor of monocarboxylate transporters completely inhibited mitochondrial respiration in all tissues regardless of substrate. Collectively, these data suggest D-lactate inhibition of pyruvate and L-lactate mitochondrial utilization may be due, in part, to competitive inhibition of the monocarboxylate transporters responsible for the transport of pyruvate and lactate into the mitochondria. Since mitochondrial oxidative phosphorylation is the main source of ATP production in various tissues, disruption of mitochondrial respiratory function in brain and heart may compromise cellular energy status and result in toxicity. Hence, D-lactate mediated reductions in mitochondrial energy production may contribute to the neurological and cardiac toxicity associated with D-lactic acidosis. L-Lactic acidosis would not result in a cellular energy deficiency due to LDH mediated conversion of L-lactate to pyruvate by liver, brain, and heart mitochondria. Further investigation is warranted to determine the relationship between reductions in mitochondrial energy production to cellular energy deficiency and organ dysfunction.

\section{Acknowledgements}

This work was funded by Natural Sciences and Engineering Research Council of Canada (NSERC).

\section{Author details}

'College of Pharmacy and Nutrition, University of Saskatchewan, Saskatoon, SK, Canada. ${ }^{2}$ Large Animal Clinical Sciences, Western College of Veterinary Medicine, Saskatoon, SK, Canada.

\section{Authors' contributions}

All authors contributed substantially to the body of work and have read and approved the final submitted manuscript.

\section{Competing interests}

The authors declare that they have no competing interests.

Received: 6 October 2011 Accepted: 1 February 2012

Published: 1 February 2012

\section{References}

1. Kondoh $\mathrm{Y}$, Kawase M, Kawakami Y, Ohmori S: Concentrations of D-lactate and its related metabolic intermediates in liver, blood, and muscle of diabetic and starved rats. Res Exp Med (Berl) 1992, 192:407-414.

2. Dahhak S, Uhlen S, Mention K, Romond MB, Fontaine M, Gottrand F, Turck D, Michaud L: D-lactic acidosis in a child with short bowel syndrome. Arch Pediatr 2008, 15:145-148.

3. Lorenz I: D-Lactic acidosis in calves. Vet J 2009, 179:197-203.

4. Weizman Z, Houri S, Ben-Ezer Gradus D: Type of acidosis and clinical outcome in infantile gastroenteritis. J Pediatr Gastroenterol Nutr 1992, 14:187-191.

5. Ewaschuk JB, Naylor JM, Palmer R, Whiting SJ, Zello GA: D-lactate production and excretion in diarrheic calves. J Vet Intern Med 2004, 18:744-747.

6. Lorenz I, Gentile A, Klee W: Investigations of D-lactate metabolism and the clinical signs of D-lactataemia in calves. Vet Rec 2005, 156:412-415.

7. Abeysekara S, Naylor J, Wassef A, Isak U, Zello G: D-Lactic acid-induced neurotoxicity in a calf model. Am J Physiol Endocrinol Metab 2007, 293 E558.

8. Sheedy JR, Wettenhall RE, Scanlon D, Gooley PR, Lewis DP, McGregor N, Stapleton DI, Butt HL, KL DEM: Increased d-lactic Acid intestinal bacteria in patients with chronic fatigue syndrome. In Vivo 2009, 23:621-628.

9. Talasniemi JP, Pennanen S, Savolainen H, Niskanen L, Liesivuori J: Analytical investigation: assay of D-lactate in diabetic plasma and urine. Clin Biochem 2008, 41:1099-1103.

10. Lu J, Zello GA, Randell E, Adeli K, Krahn J, Meng QH: Closing the anion gap: contribution of D-lactate to diabetic ketoacidosis. Clin Chim Acta 2011, 412:286-291.

11. Cross S, Callaway C: D-Lactic acidosis and selected cerebellar ataxias. Mayo Clin Proc 1984, 59:202-205.

12. Bolanos JP, Almeida A, Moncada S: Glycolysis: a bioenergetic or a survival pathway? Trends Biochem Sci 2010, 35:145-149.

13. Huss JM, Kelly DP: Mitochondrial energy metabolism in heart failure: a question of balance. J Clin Invest 2005, 115:547-555.

14. Beal MF, Hyman BT, Koroshetz W: Do defects in mitochondrial energy metabolism underlie the pathology of neurodegenerative diseases? Trends Neurosci 1993, 16:125-131.

15. Ereci ska M, Wilson D: Regulation of cellular energy metabolism. J Membr Biol 1982, 70:1-14.

16. Leverve XM: Mitochondrial function and substrate availability. Crit Care Med 2007, 35:5454-460.

17. Brooks GA, Brown MA, Butz CE, Sicurello JP, Dubouchaud H: Cardiac and skeletal muscle mitochondria have a monocarboxylate transporter MCT1. J Appl Physiol 1999, 87:1713-1718.

18. Gladden LB: Lactate metabolism: a new paradigm for the third millennium. J Physiol 2004, 558:5-30.

19. Passarella $S$, de Bari L, Valenti D, Pizzuto R, Paventi G, Atlante A: Mitochondria and L-lactate metabolism. FEBS lett 2008, 582:3569-3576.

20. Smith D, Pernet A, Hallett WA, Bingham E, Marsden PK, Amiel SA: Lactate: a preferred fuel for human brain metabolism in vivo. $J$ Cereb Blood Flow Metab 2003, 23:658-664. 
21. Lemire J, Mailloux RJ, Appanna VD: Mitochondrial lactate dehydrogenase is involved in oxidative-energy metabolism in human astrocytoma cells (CCF-STTG1). PLoS One 2008, 3:e1550.

22. Wang Q, Lu Y, Yuan M, Darling IM, Repasky EA, Morris ME: Characterization of monocarboxylate transport in human kidney HK-2 cells. Mol Pharm 2006, 3:675-685.

23. Poole R, Cranmer S, Halestrap A, Levi A: Substrate and inhibitor specificity of monocarboxylate transport into heart cells and erythrocytes. Further evidence for the existence of two distinct carriers. Biochem J 1990, 269:827.

24. Ros J, Pecinska N, Alessandri B, Landolt H, Fillenz M: Lactate reduces glutamate induced neurotoxicity in rat cortex. J Neurosci Res 2001, 66:790-794.

25. Petersen C: D-lactic acidosis. Nutr Clin Pract 2005, 20:634-645

26. Flick MJ, Konieczny SF: Identification of putative mammalian D-lactate dehydrogenase enzymes. Biochem Biophys Res Commun 2002, 295:910-916.

27. Tubbs PK: The metabolism of D-alpha-hydroxy acids in animal tissues. Ann N Y Acad Sci 1965, 119:920-926.

28. Gibbs ME, Hertz L: Inhibition of astrocytic energy metabolism by Dlactate exposure impairs memory. Neurochem Int 2008, 52:1012-1018.

29. Schneider WC: Intracellular distribution of enzymes; the oxidation of octanoic acid by rat liver fractions. J Biol Chem 1948, 176:259-266.

30. Johnson D, Lardy H: Isolation of liver or kidney mitochondria. Methods Enzymol 1967, 10:94-96.

31. Nedergaard J, Cannon B: Overview-Preparation and properties of mitochondria from different sources. Methods Enzymol 1979, 55:3-28.

32. Kowaltowski AJ, Castilho RF, Grijalba MT, Bechara EJH, Vercesi AE: Effect of Inorganic Phosphate Concentration on the Nature of Inner Mitochondrial Membrane Alterations Mediated by Ca lons. J Biol Chem 1996, 271:2929.

33. Gornall AG, Bardawill CJ, David MM: Determination of serum proteins by means of the biuret reaction. J biol Chem 1949, 177:751-766.

34. de Bari L, Atlante A, Guaragnella N, Principato G, Passarella S: D-Lactate transport and metabolism in rat liver mitochondria. Biochem J 2002, 365:391-403.

35. Cadenas $\mathrm{E}$, Davies $\mathrm{K}$ : Mitochondrial free radical generation, oxidative stress, and aging1. Free Radic Biol Med 2000, 29:222-230.

36. Maitra PK, Estabrook RW: Studies of baker's yeast metabolism. II. The role of adenine nucleotides and inorganic phosphate in the control of respiration during alcohol oxidation. Arch Biochem Biophys 1967, 121:129-139.

37. Estabrook RW: Mitochondrial respiratory control and the polarographic measurement of ADP: O ratios. Methods Enzymol 1967, 10:41-47.

38. Chelstowska A, Liu Z, Jia Y, Amberg D, Butow RA: Signalling between mitochondria and the nucleus regulates the expression of a new Dlactate dehydrogenase activity in yeast. Yeast 1999, 15:1377-1391.

39. Oh MS, Uribarri J, Alveranga D, Lazar I, Bazilinski N, Carroll HJ: Metabolic utilization and renal handling of D-lactate in men. Metabolism 1985, 34:621-625.

40. Christopher MM, Broussard JD, Fallin CW, Drost NJ, Peterson ME: Increased serum D-lactate associated with diabetic ketoacidosis. Metabolism 1995, 44:287-290.

41. Chan L, Slater J, Hasbargen J, Herndon DN, Veech RL, Wolf S: Neurocardiac toxicity of racemic D, L-lactate fluids. Integr Physiol Behav Sci 1994, 29:383-394.

42. Hoffmann GF, Zschocke J, Nyhan WL: Inherited metabolic diseases: a clinical approach Springer Verlag; 2009.

43. Lehninger AL: The Mitochondrion New York: W. A. Benjamin; 1964, 135

44. Higgins ES: Factors influencing respiratory control in brain mitochondria. J Neurochem 1968, 15:589-596.

45. Chang GG, Tong $L$ : Structure and function of malic enzymes, a new class of oxidative decarboxylases. Biochemistry 2003, 42:12721-12733.

46. Chance B, Williams GR: Respiratory enzymes in oxidative phosphorylation. I. Kinetics of oxygen utilization. J Biol Chem 1955, 217:383-393.

47. Messer Jl, Jackman MR, Willis WT: Pyruvate and citric acid cycle carbon requirements in isolated skeletal muscle mitochondria. Am J Physiol Cell Physiol 2004, 286:C565-572.

48. Brooks GA, Dubouchaud H, Brown M, Sicurello JP, Butz CE: Role of mitochondrial lactate dehydrogenase and lactate oxidation in the intracellular lactate shuttle. Proc Natl Acad Sci USA 1999, 96:1129-1134.
49. Fleischer S, Rouser G, Fleischer B, Casu A, Kritchevsky G: Lipid composition of mitochondria from bovine heart, liver, and kidney. J Lipid Res 1967, 8:170.

50. Chance B, Williams GR: The respiratory chain and oxidative phosphorylation. Adv Enzymol Relat Subj Biochem 1956, 17:65-134.

51. Poole RC, Cranmer SL, Halestrap AP, Levi AJ: Substrate and inhibitor specificity of monocarboxylate transport into heart cells and erythrocytes. Further evidence for the existence of two distinct carriers. Biochem J 1990, 269:827-829.

52. Kline ES, Brandt RB, Laux JE, Spainhour SE, Higgins ES, Rogers KS, Tinsley SB, Waters MG: Localization of L-lactate dehydrogenase in mitochondria. Arch Biochem Biophys 1986, 246:673-680.

53. Szczesna-Kaczmarek A: L-lactate oxidation by skeletal muscle mitochondria. Int J Biochem 1990, 22:617-620.

\section{doi:10.1186/1743-7075-9-6}

Cite this article as: Ling et al:: D-Lactate altered mitochondrial energy production in rat brain and heart but not liver. Nutrition \& Metabolism 2012 9:6.

\section{Submit your next manuscript to BioMed Central and take full advantage of:}

- Convenient online submission

- Thorough peer review

- No space constraints or color figure charges

- Immediate publication on acceptance

- Inclusion in PubMed, CAS, Scopus and Google Scholar

- Research which is freely available for redistribution

Submit your manuscript at www.biomedcentral.com/submit
C Biomed Central 